\title{
Proximal femoral head transcriptome reveals novel candidate genes related to epiphysiolysis in broiler chickens
}

Jane de Oliveira Peixoto ${ }^{1,2}$, Igor Ricardo Savoldi ${ }^{1,3,4}$, Adriana Mércia Guaratini Ibelli, ${ }^{1,2,3}$, Maurício Egídio Cantão ${ }^{1}$, Fátima Regina Ferreira Jaenisch ${ }^{1}$, Poliana Fernanda Giachetto ${ }^{5}$, Matthew Lee Settles ${ }^{6}$, Ricardo Zanella, ${ }^{7,8}$, Jorge Augusto Petroli Marchesi ${ }^{1,9}$, José Rodrigo Pandolfi ${ }^{1}$, Luiz Lehmann Coutinho ${ }^{10}$ and Mônica Corrêa Ledur ${ }^{1,4^{*}}$ (D)

\begin{abstract}
Background: The proximal femoral head separation (FHS) or epiphysiolysis is a prevalent disorder affecting the chicken femur epiphysis, being considered a risk factor to infection which can cause bacterial chondronecrosis with osteomyelitis in broilers. To identify the genetic mechanisms involved in epiphysiolysis, differentially expressed (DE) genes in the femur of normal and FHS-affected broilers were identified using RNA-Seq technology. Femoral growth plate (GP) samples from 35-day-old commercial male broilers were collected from 4 healthy and 4 FHS-affected broilers. Sequencing was performed using an Illumina paired-end protocol. Differentially expressed genes were obtained using the edgeR package based on the False Discovery Rate (FDR < 0.05).

Results: Approximately 16 million reads/sample were generated with $2 \times 100$ bp paired-end reads. After data quality control, approximately 12 million reads/sample were mapped to the reference chicken genome (Galgal5). A total of 12,645 genes were expressed in the femur GP. Out of those, 314 were DE between groups, being 154 upregulated and 160 downregulated in FHS-affected broilers. In the functional analyses, several biological processes (BP) were overrepresented. Among them, those related to cell adhesion, extracellular matrix (ECM), bone development, blood circulation and lipid metabolism, which are more related to chicken growth, are possibly involved with the onset of FHS. On the other hand, BP associated to apoptosis or cell death and immune response, which were also found in our study, could be related to the consequence of the FHS.

Conclusions: Genes with potential role in the epiphysiolysis were identified through the femur head transcriptome analysis, providing a better understanding of the mechanisms that regulate bone development in fast-growing chickens. In this study, we highlighted the importance of cell adhesion and extracellular matrix related genes in triggering FHS. Furthermore, we have shown new insights on the involvement of lipidemia and immune response/ inflammation with FHS in broilers. Understanding the changes in the GP transcriptome might support breeding strategies to address poultry robustness and to obtain more resilient broilers.
\end{abstract}

Keywords: BCO, Femoral head necrosis, Femur growth plate, RNA-sequencing, Leg problems

\footnotetext{
* Correspondence: monica.ledur@embrapa.br

'Embrapa Suínos e Aves, Rodovia BR-153, Km 110, Distrito de Tamanduá,

Caixa Postal: 321, Concórdia, Santa Catarina 89715-899, Brazil

${ }^{4}$ Programa de Pós-Graduação em Zootecnia, UDESC-Oeste, Chapecó, SC,

Brazil

Full list of author information is available at the end of the article
}

(c) The Author(s). 2019 Open Access This article is distributed under the terms of the Creative Commons Attribution 4.0 International License (http://creativecommons.org/licenses/by/4.0/), which permits unrestricted use, distribution, and reproduction in any medium, provided you give appropriate credit to the original author(s) and the source, provide a link to the Creative Commons license, and indicate if changes were made. The Creative Commons Public Domain Dedication waiver (http://creativecommons.org/publicdomain/zero/1.0/) applies to the data made available in this article, unless otherwise stated. 


\section{Background}

The poultry meat industry is extraordinarily efficient in supplying high-quality and affordable food for human and animal consumption. Chicken is one of the main sources of animal protein for humans, and nowadays is the second most widely consumed meat in the world [1]. In the last decades, the improvement in poultry genetic potential was remarkable, and was the major factor for rapid growth and feed efficiency [2, 3]. As a consequence, the modern broilers have experienced huge phenotypic and genetic changes. Chicken production efficiency contributes to reducing environmental impact, as demonstrated by the smaller water footprint compared with other livestock production [4]. However, the genetic progress in production traits was concomitant with the appearance of undesirable effects, including increased skeletal defects, metabolic disorders and altered immune function [5].

Zuidhof et al. [3] showed that broiler growth rate has increased by over $400 \%$ with a concomitant $50 \%$ reduction in feed conversion ratio from 1957 to 2005, when comparing representative lines of those years in identical environments. The metabolism has increased in meattype chickens due to the intense selection for growth rate and the intensification of the production system; as a consequence, metabolic disorders became a worldwide concern, causing more economic losses than infectious agents [6]. These conditions affect primarily the cardiovascular and musculoskeletal systems [6] and the incidence of bone problems is nowadays considered one of the main concerns to the poultry industry due to the significant economic losses and by severely compromising poultry welfare $[6,7]$. Moreover, leg disorders are one of the major welfare problems in the poultry production system [8]. Severe leg disorders may compromise the broilers welfare due to pain and inability to walk, leading to feed and water restriction [7]. In addition, locomotor disorders violates four of the five freedoms that farmed animals are expected to enjoy according to the Farm Animal Welfare Council [9]. These problems have a high prevalence in the farms, ranging from 9 to $33 \%[10,11]$. The proximal femoral head is highly exposed to injury under strenuous conditions, especially in broilers. Femur and tibia growth plates are sites of huge mechanical stress [12] and the increase in body weight significantly alters the femur center of gravity [13].

A common disorder affecting the proximal femur epiphysis is the proximal femoral head separation or epiphyseolysis (FHS), which is the separation of the growth plate (GP) from the articular cartilage (AC) resulting in disarticulation of the coxofemoral joint $[13,14]$. Durairaj et al. [15] consider FHS as a metabolic skeletal problem in fast-growing chickens that increases the femoral epiphysis vulnerability to separation and is implicated in subsequent problems, such as bacterial infection [6]. The real incidence of FHS in broiler chickens is difficult to estimate because the lesion remains subclinical and the etiologic basis of FHS in young poultry is not completely understood [13].

The FHS is a predisposing factor for infection and osteomyelitis [15], which are conditions related to the Bacterial Chondronecrosis with Osteomyelitis (BCO), also known as Femoral Head Necrosis (FHN) [16]. The $\mathrm{BCO}$ is considered the most important leg disorder and the main cause of lameness in commercial broiler flocks, mainly affecting heavy birds after 35 days of age [7, 12, $16,17]$. In BCO affected flocks, morbidity is around $15 \%$, which results in significant losses $[16,18]$. Therefore, in order to investigate the molecular events taking place during the onset and development of the epiphysiolysis in fast-growing chickens, the present study used the RNA-Seq technology to identify differentially expressed genes in the proximal femur head of normal and FHS-affected broilers.

\section{Results}

Transcriptome analysis

High-throughput RNA sequencing was used to generate a whole characterization of the proximal femur GP transcriptome of health and FHS-affected broilers. The multi-dimensional scaling (MDS) plot provided a spatial representation of data (Additional file 1) grouping samples in distinct clusters. It was possible to observe the homogeneity between samples from each group, which was also confirmed in the Heatmap plot (Fig. 1). The cluster analysis with the DE genes revealed a clear separation between FHS-affected and unaffected samples, suggesting homogeneity of the clinical classification of broilers in healthy and FHS-affected (Fig. 1).

Approximately, 16 million reads/sample were generated with $2 \times 100 \mathrm{bp}$ paired-end reads. After data quality control, in average, about 12 million reads/sample were mapped to the chicken reference genome (Galgal5, Ensembl release 87). The average reads mapped across samples was $98.99 \%$ and a total of 12,645 genes were expressed in the GP transcriptome. Out of those, 314 were DE between normal and FHS-affected broilers (FDR < 0.05), being 154 upregulated and 160 downregulated (Additional file 2) in the affected compared to normal group. The top 10 down and upregulated transcripts are shown in Table 1.

\section{Functional analyses}

The gene ontology analysis performed with the DE genes in Blast2GO showed that 290 annotated genes were enriched in 19 biological process (Fig. 2). According to the GO molecular function (Fig. 3), most of the DE genes presented binding functions, such as binding to 

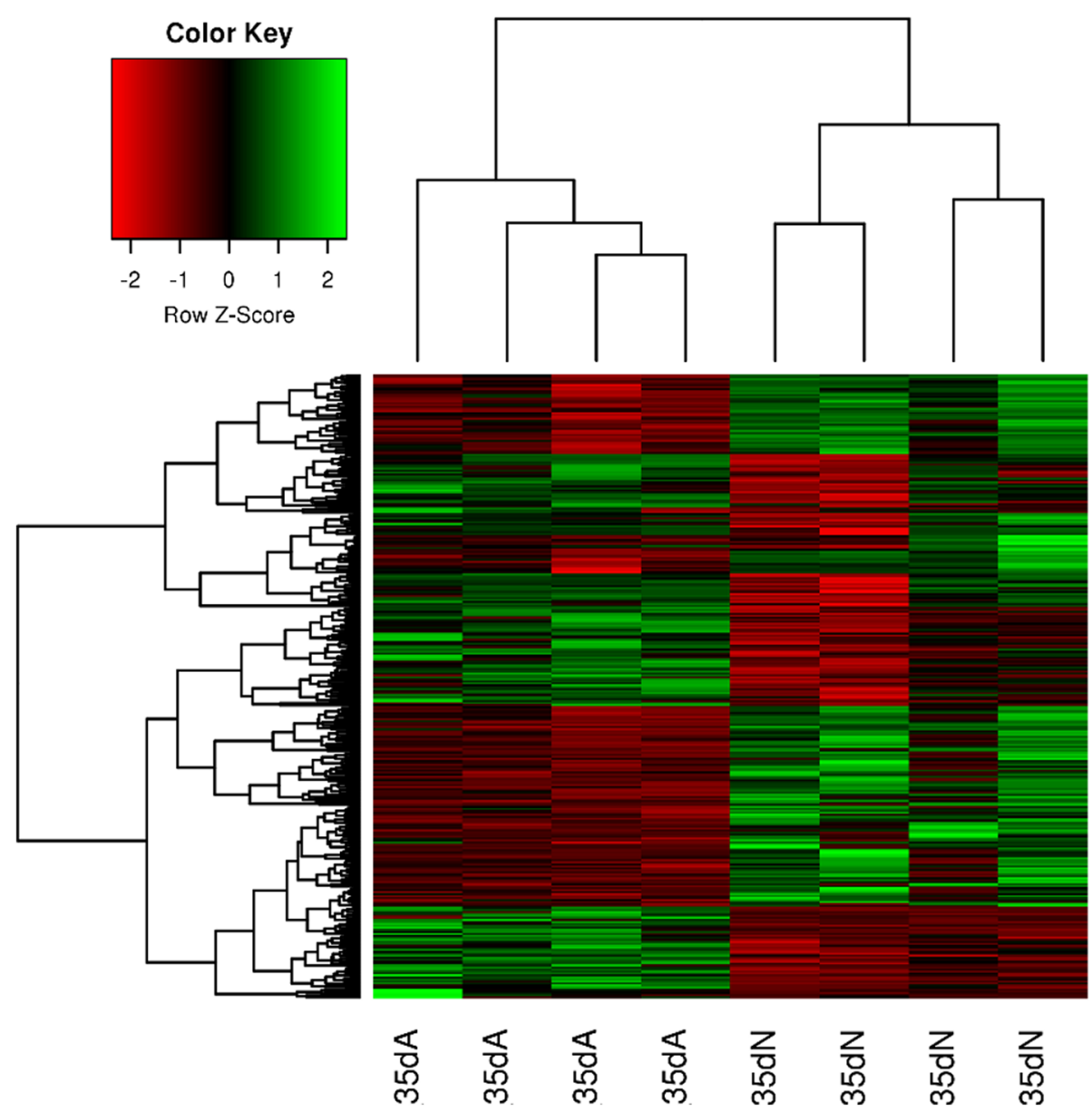

Fig. 1 Gene cluster classification for FHS-affected and unaffected samples, with the 314 DE genes. In the heatmap, the expression for each gene is shown in the rows and samples are visualized in the columns, showing a hierarchical clustering of genes and samples. Genes are upregulated (in red) and downregulated (in green) in the affected samples

ions, protein, heterocyclic compound and organic cyclic compound. Furthermore, transferase and hydrolase activity were also well represented molecular functions of the DE genes in the growth plate tissue (Fig. 3).

Gene ontology analysis was also performed in DAVID database, finding a total of 226 BP (Additional file 3), which were summarized in 9 superclusters with the REViGO tool (Fig. 4), including the following BP: response do endogenous stimulus, tissue development, blood circulation, inorganic ion homeostasis, immune effector process, cytokine metabolism and cell adhesion.

A strong evidence of interaction of several DE genes was observed in the gene network analysis (Fig. 5). A central group of genes was found, such as JAK2, PDGFRA, STAT5A, PDGFRL and MAPK12 (ENSGA LG00000019384) that are mostly involved in blood circulation and angiogenesis (Fig. 5). These genes were also connected with many genes related to bone differentiation (i.e. OGN, $A D I P O Q, G L I 2$ ), extracellular matrix (i.e. collagen genes, FN1), tissue homeostasis (i.e. JAK2,
IL7R, RHAG, EPB42) and immune response (i.e. IL7R, IRF1, JAK2) (Fig. 5). Other 5 small branch of genes were found, with no direct node with the main group of genes, being: 1); PLCB2, ITPKA, ITPK1 and ITPR3; 2) OSBPL1A, ITIH5, C1QTNFB and GIMAP5; 3) H1F0, TRIM23, TUBB1, KIF26B, KIF1A and SMAP2; 4) PNPLA2, ACSL6, SCD, ALDH1A1, DDC and ALDH6A1 and 5) NMNAT1, NT5C3, ADA, CECR1, AMPD3 and $B T B D 10$ (Fig. 5). Nevertheless, some of those genes could be considered hubs since they are involved in the expression of several other genes (Fig. 6).

It is possible to observe that cell adhesion is one of the most enriched BP, grouping genes that interact with angiogenesis, muscle development, skeletal systems, immune response and lipid metabolism (Fig. 7).

\section{Validation by quantitative PCR}

In the $\mathrm{qPCR}$ analysis, five of the six analyzed genes (LRP1B, Col28A, PERP2, FAM180A and CHST1) were differentially expressed $(P<0.05)$ in the proximal femur 
Table 1 Top 10 down and upregulated genes in the proximal femur transcriptome of FHS-affected compared to healthy broilers, according to the LogFC and FDR

\begin{tabular}{llll}
\hline Ensembl ID & LogFC & FDR & Gene \\
\hline ENSGALG00000027184 & -5.38 & $5.00 \mathrm{E}-10$ & OLFML1 \\
ENSGALG00000032856 & -4.92 & $1.07 \mathrm{E}-06$ & GFRA2 \\
ENSGALG00000039376 & -4.88 & $7.40 \mathrm{E}-07$ & DPT \\
ENSGALG00000024428 & -4.84 & $2.39 \mathrm{E}-06$ & C17orf67 \\
ENSGALG00000028459 & -4.46 & $1.18 \mathrm{E}-07$ & FAM180A \\
ENSGALG00000015307 & -4.40 & $2.19 \mathrm{E}-07$ & ABI3BP \\
ENSGALG00000017191 & -3.81 & $2.35 \mathrm{E}-04$ & ANGPTL5 \\
ENSGALG00000043703 & -3.47 & $1.26 \mathrm{E}-07$ & \\
ENSGALG00000032220 & -3.27 & $8.77 \mathrm{E}-07$ & ELN \\
ENSGALG000000016511 & -3.14 & $7.71 \mathrm{E}-03$ & ADGRG2 \\
ENSGALG00000009479 & 2.20 & $8.76 \mathrm{E}-03$ & SAMD9L \\
ENSGALG00000044811 & 2.21 & $3.60 \mathrm{E}-05$ & MSMB-like \\
ENSGALG00000046192 & 2.21 & $5.45 \mathrm{E}-04$ & CCL19 \\
ENSGALG000000026727 & 2.25 & 0.0295 & SLC30A10 \\
ENSGALG00000043860 & 2.42 & 0.0030 & PGR2 \\
ENSGALG00000023933 & 2.42 & 0.024 & GOS2 \\
ENSGALG00000043254 & 2.56 & $1.11 \mathrm{E}-03$ & EPX \\
ENSGALG00000028256 & 2.67 & $3.58 \mathrm{E}-03$ & CCL19 \\
ENSGALG00000023622 & 3.02 & $7.06 \mathrm{E}-03$ & AVD \\
ENSGALG00000032614 & 3.37 & $1.51 \mathrm{E}-04$ & CHAT2 \\
\hline OFGI OFaCom & &
\end{tabular}

OLFML1 Olfactomedin like 1, GFRA2 GDNF family receptor alpha 2, DPT Dermatopontin, C17orf67 Chromosome 17 open reading frame 67 orf67, FAM180A Family with sequence similarity 180 member $A, A B I 3 B P A B I$ family member 3 binding protein, ANGPTL5 Angiopoietin like 5, ADGRG2 Adhesion G protein-coupled receptor G2, SAMD9L Sterile alpha motif domain containing 9like, SLC30A10 Solute carrier family 30 member, GOS2GO/G1 switch 2, CCL19:CC:motif chemokine ligand 19, AVD Avidin, CHAT2 ADP-ribosyltransferase. ${ }^{a}$ Genes annotated in the Ensembl 98 release. ELN: Elastin, MSMB-like:

Microseminoprotein Beta Like, CCL19 C-C Motif Chemokine Ligand 19, PGR2 Progesterone receptor 2, EPX Eosinophil Peroxidase
GP between normal and FHS-affected groups. Furthermore, all of these genes kept the same expression profile observed in the RNA-Seq analysis (Table 2, Fig. 8), confirming the results from our RNA-Seq study. Although the PLIN1 was not DE between groups in the qPCR analysis, it had similar expression levels between the two studies, following the same pattern of upregulation in FHS-affected broilers. The difference between the statistical significance from the two approaches could be due to the heterogeneity of the expression among samples within groups.

\section{Discussion}

The selection of broiler chickens for rapid growth and increased muscle yield has resulted in some undesirable consequences including a high incidence of skeletal problems [15]. Separation of the femoral epiphysis from the articular cartilage (FHS) may be a metabolic problem that leads the femur vulnerable to other anomalies. The endochondral ossification of long bones consists of the proliferation and differentiation of chondrocytes in the epiphysial growth plate [19]. Although the ossification is a conserved process across several mammal species, chickens present some differences in bone physiology, including fast bone remodeling [20]. In the growing phase, femur and tibia have a very fast development [21], and they continue to grow until the adult age. This pattern has been observed by several authors studying the rapid leg bone growth in broilers [22-24]. Moreover, it has been a consensus that the skeleton does not mature fast enough to support the growth potential of modern broilers $[18,25]$ and that femur and tibia growth plates are sites of huge mechanical stress, especially due

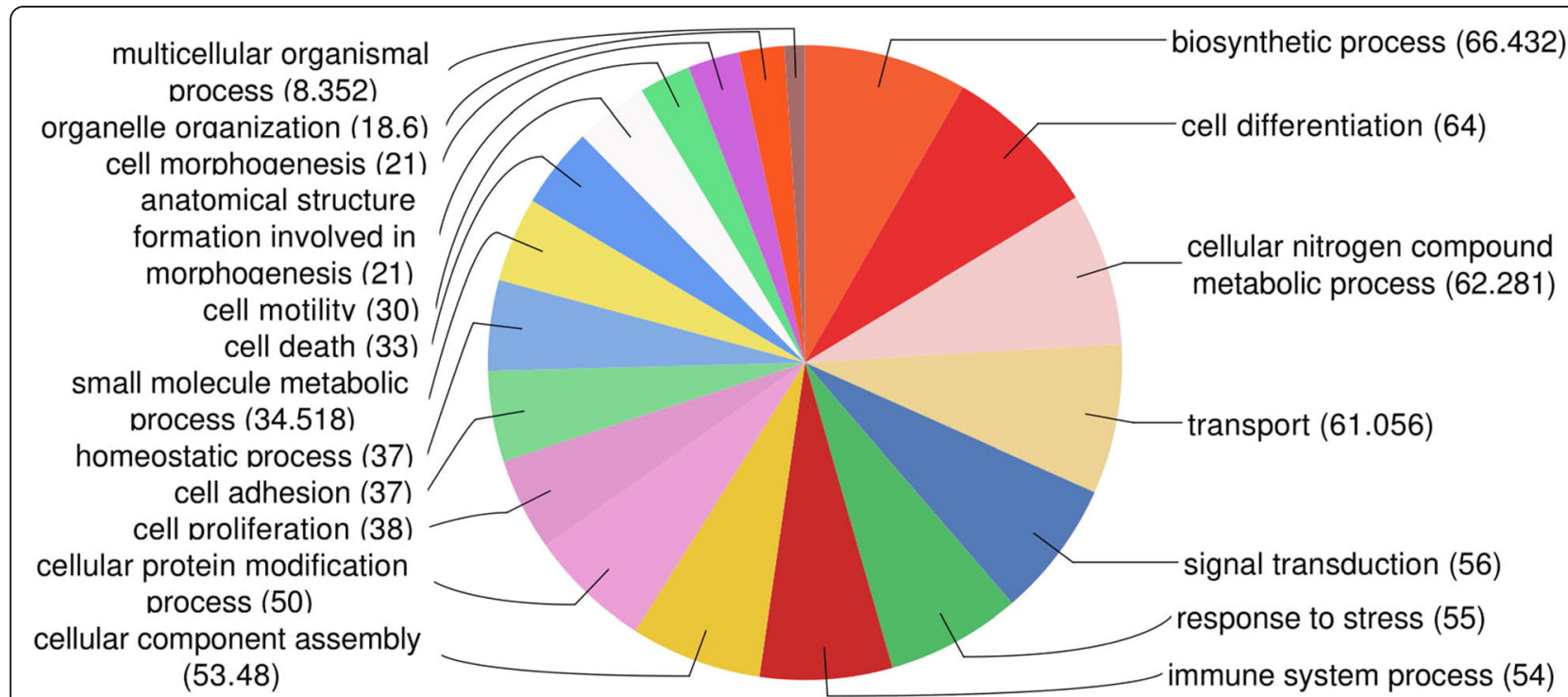

Fig. 2 Distribution of DE genes according to the gene ontology category of biological processes in Blast2GO analysis 


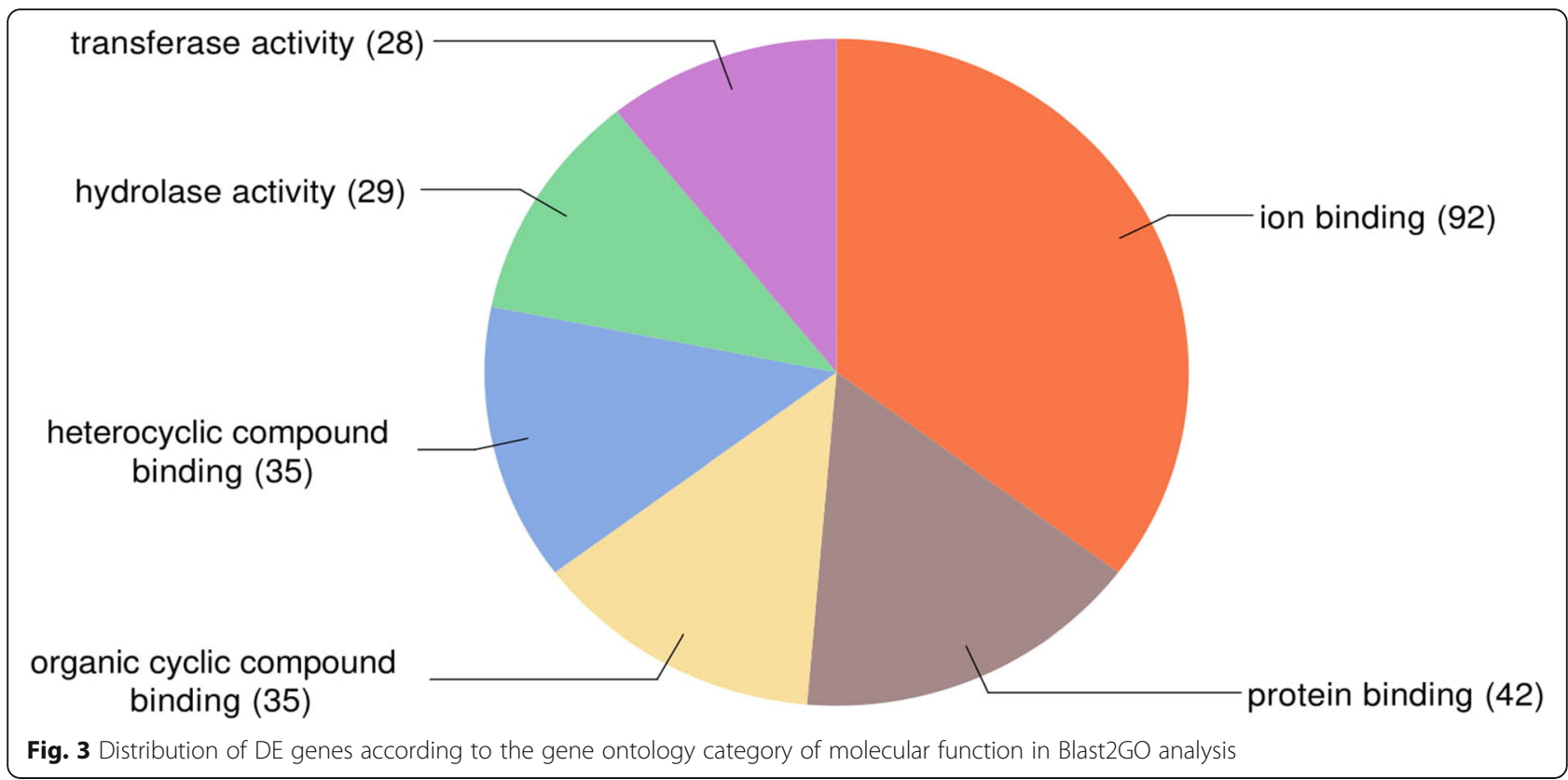

to the high growth rates [12]. In this sense, the increased metabolism and mechanical stressful condition are predisposing factors to some of the most important leg problems in the femur and tibia growth plates, such as FHS, BCO and tibial dyschondroplasia (TD). Therefore, the global transcriptome analyses of the femur growth plate tissue in the proximal head can unravel regulatory pathways that control the development of skeletal abnormalities related to FHS in chickens.

Here, we provided the first FHS-related transcriptome and identified a group of genes involved with FHS in broilers. These findings are an important

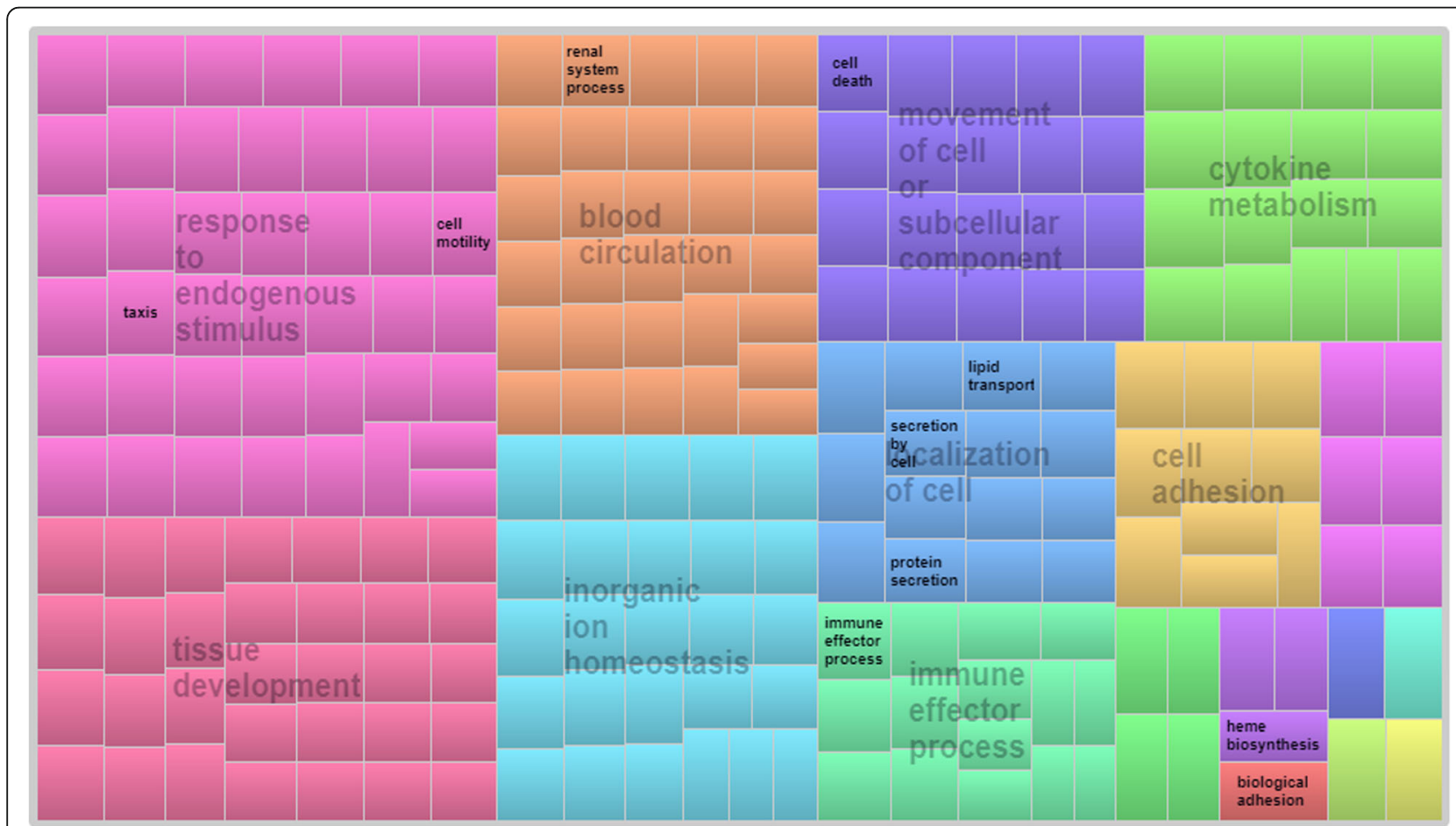

Fig. 4 Superclusters of biological process enriched for up and downregulated genes related to FHS. Different colors show different superclusters and the size of each box is determined by the uniqueness of the categories 


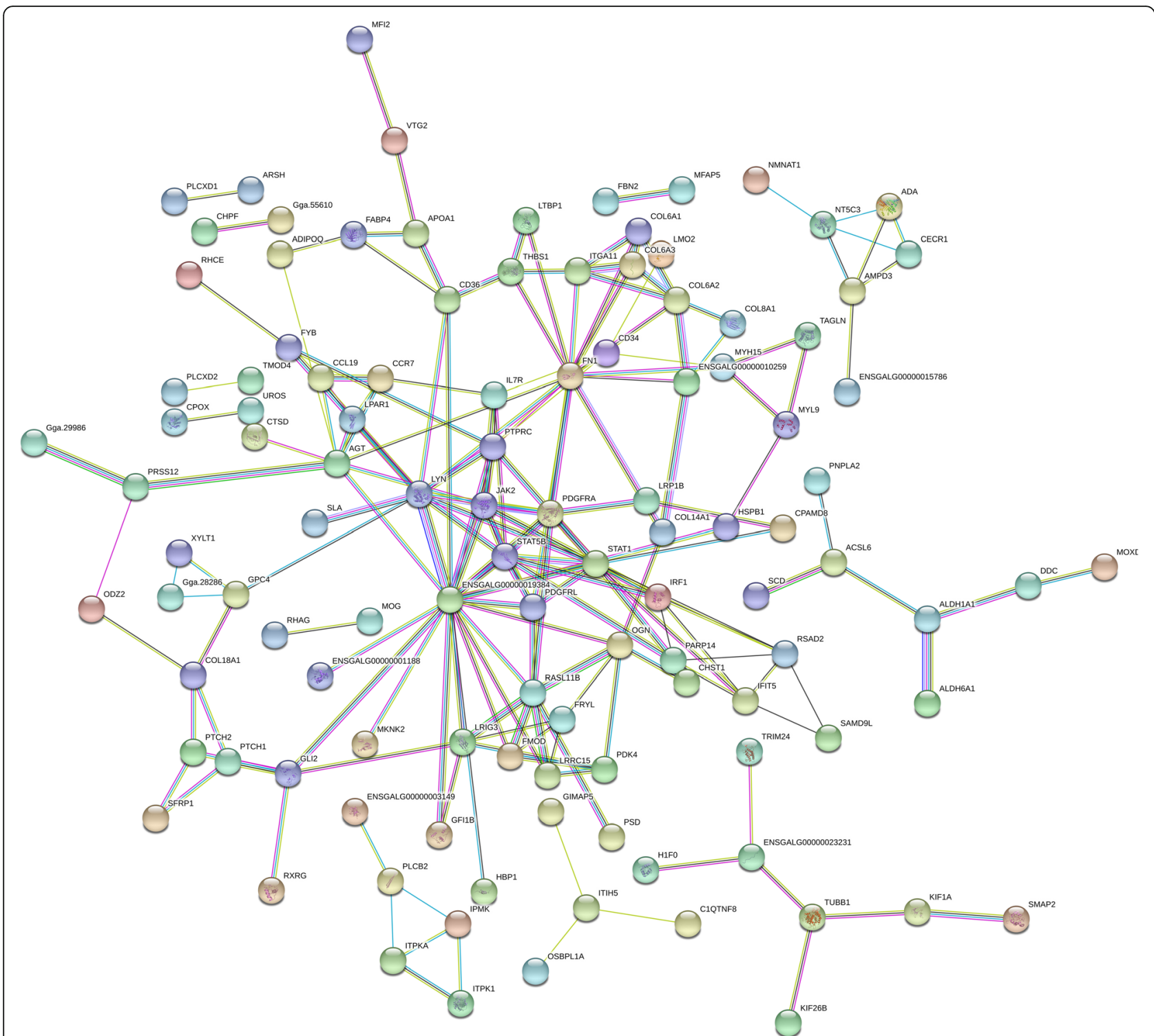

Fig. 5 Gene network of differentially expressed genes related to femoral head separation in broilers using Stringdb. Nodes indicate the number of predicted gene interactions

resource of functional candidate genes for future studies on bone development in fast-growing chickens. The main BP potentially involved in triggering FHS were those related to chicken growth, such as bone development, extracellular matrix organization, lipid metabolism, blood circulation and cell adhesion (Figs. 5 and 6). On the other hand, BP associated to apoptosis or cell death and immune response processes, also found in our study, could be a consequence of the disruption on growth (Additional file 3, Fig. 7). The main BP related to physiological mechanisms involved in FHS are discussed below.
Bone development, extracellular matrix organization and cell adhesion

In broilers, 35 days of age is an important phase of the femur development during a commercial growing period [26]. At this age, the femur length as a function of body weight reaches a plateau, differing from the tibia bone that continues to lengthen [21]. Some authors indicate that most cases of $\mathrm{BCO}$, considered a progression of FHS, develop after 35 days of age [12, 16, 17], possibly because the body weight increases faster than the femur development [21]. Furthermore, it has been observed that at early ages, 0 to 7 days, fast-growing broilers have 


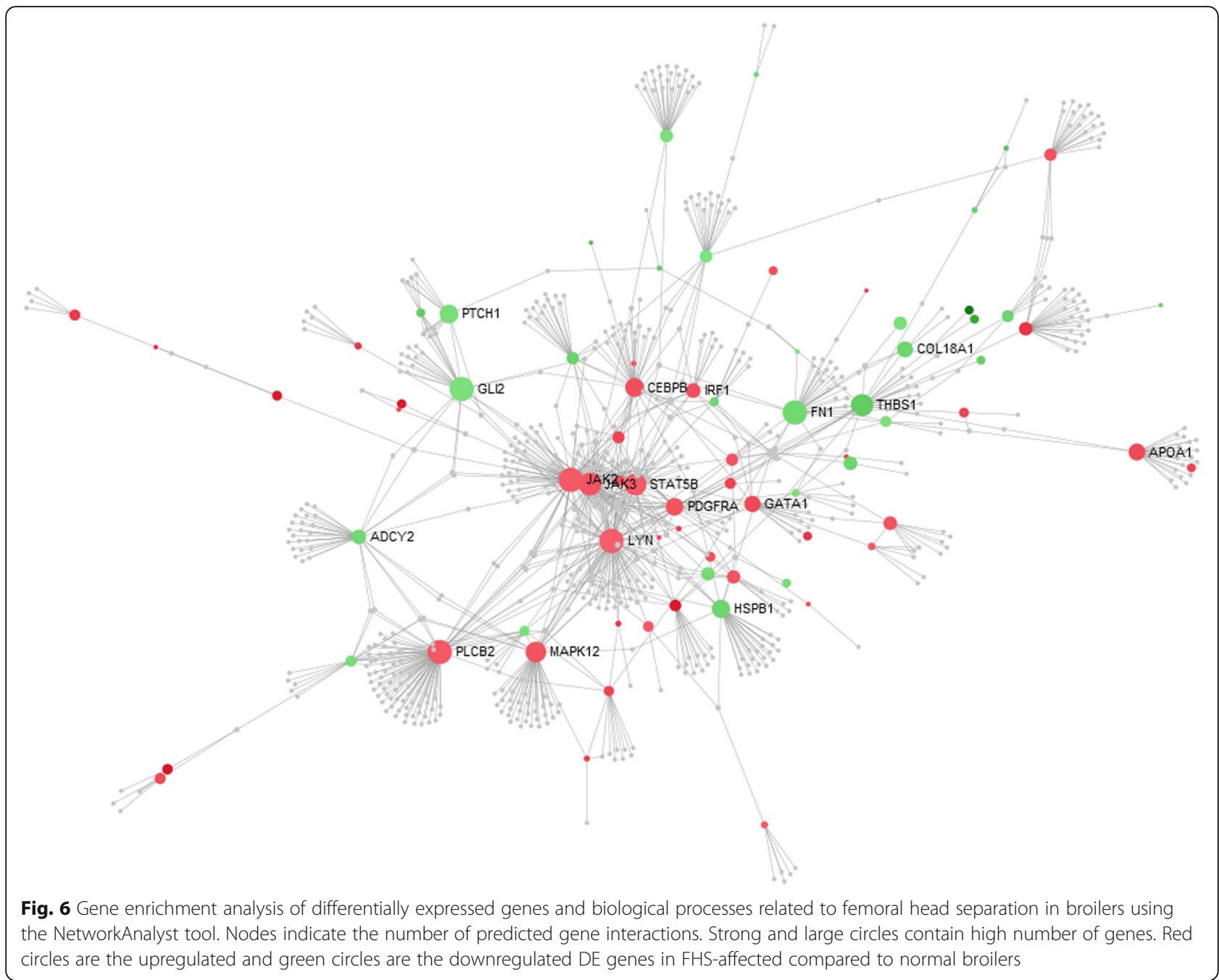

higher mean volumetric bone mineral density and heavier and longer bone than medium-growing chickens, which could indicate that bone development is adequate in the early life or even during embryogenesis in fastgrowing broilers [26].

The epiphysiolysis has been classified as a disorder related to the growing phase and a large number of $\mathrm{DE}$ genes in this study were associated to BP related to skeletal system development, ossification, osteoblast differentiation, cell development, and others (Additional file 3 , Fig. 7). These BP are directly correlated with bone function, and several genes, such as $A C S L 6, A D I P O Q, B N C 2$, LOXL2, COL6A1, COL8A1, CYTL1, FAM65B, FN1, GLI2, CD36, ITGA1 and OGN showed reduced expression in the FHS-affected broilers (Additional file 3, Fig. 5).

The bone extracellular matrix is a keystone in skeletal development and a precisely regulated expression of cellular and extracellular matrix genes is required to the correct osteogenesis [27]. The ECM is composed by a proteinaceous fiber component (collagen) and an amorphous ground substance (proteoglycan) being a dynamic network of molecules secreted by cells under regulation of transcription factors, signaling molecules, hormones and local growth factors. According to Velleman [27], although the composition of the ECM is known, the function of each macromolecule components and their interaction is poorly understood and the role of the bone ECM in skeletal development has been ignored by the poultry scientific community.

Cell adhesion related BP could have a key function in triggering FHS (Fig. 4, Fig. 7), since it was the main hub to connect all of the other processes that were involved with FHS (Fig. 7). The Lysyl oxidase-like-2 (LOXL2) gene can be highlighted since it catalyzes the oxidative deamination of peptidyl lysine residues, promoting the formation of lysyl-derived crosslinks in collagens and elastin [28], genes that were also DE in our study. LOXL2-catalyzed crosslinks are essential posttranslational modifications in the biosynthesis and maintenance of the ECM, contributing to the tensile strength 


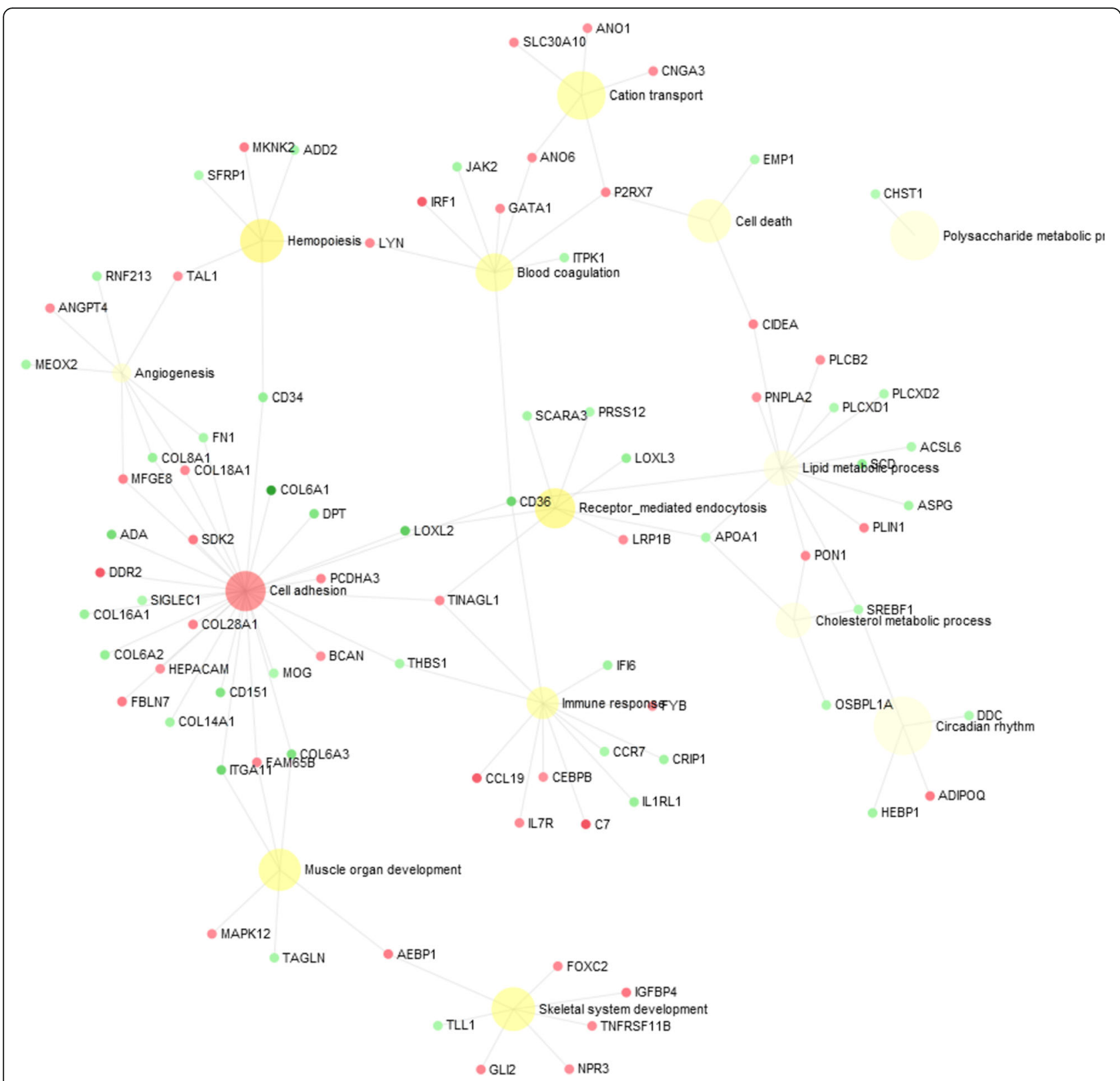

Fig. 7 Bipartite gene network of differentially expressed genes related to femoral head separation in broilers using NetworkAnalyst tool. Nodes indicate the number of predicted gene interactions. Red circles are the upregulated and green circles are the downregulated DE genes in FHSaffected compared to normal broilers. The hub genes are highlighted in green and red

and stability of tissues [29]. Therefore, the downregulation of LOXL2 and the other genes could reduce the cell adhesion, which could facilitate the separation of cartilage and bone tissues. Furthermore, this gene is also connected with immune biological responses, blood coagulation and lipid metabolism functions (Fig. 7).

Many biological processes related to ECM and also cell adhesion were enriched, such as ECM organization, glycosaminoglycan metabolic process, aminoglycan metabolic process, extracellular structure organization, connective tissue development, biological adhesion, regulation of cell adhesion, cell adhesion, cell-matrix adhesion, and others (Additional file 3), where DE genes were downregulated in FHS-broilers $(A B I 3 B P, A D I P O Q$, CD34, CD151, CHST1, COL6A1, COL6A3, COL8A1, COL16A1, CYTL1, FN1, GLI2, HSPB1, ITGA11, ITIH5, KIF26B, LOXL2, MFAP5, MYL9, PRRX1, TENM2, THBS1, TINAGL1,VIT and XYLT1) (Figs. 5, 6 and 7). Here, an important downregulated gene to be discussed is the CHST1, which participates in the glycosaminoglycan (GAGs) and aminoglycan metabolic processes involved in the ECM metabolism. The CHST1 
Table 2 Relative gene expression between healthy and FHS proximal femur in RNA-Seq and qPCR analyses in 35-day-old broilers

\begin{tabular}{|c|c|c|c|c|c|}
\hline \multirow[b]{2}{*}{ Gene } & \multicolumn{2}{|l|}{ RNA-Seq } & \multicolumn{3}{|l|}{$\mathrm{qPCR}$} \\
\hline & FDR & LogFC & LogFC & Std. error & $P$-value \\
\hline$\angle R P 1 B$ & $3.32 \mathrm{E}-05$ & -1.98 & -13.51 & $0.01-0.88$ & 0.012 \\
\hline Col28A1 & $6.24 \mathrm{E}-08$ & -2.59 & -3.62 & $0.07-1.26$ & 0.013 \\
\hline PERP2 & 1.49E-13 & -3.04 & -16.10 & $0.01-0.37$ & 0.001 \\
\hline FAM180A & $1.18 \mathrm{E}-07$ & -4.46 & -2.85 & $0.08-1.41$ & 0.035 \\
\hline CHST1 & $1.81 \mathrm{E}-05$ & -2.13 & -3.70 & $0.04-1.55$ & 0.046 \\
\hline PLIN1 & 0.03 & 2.08 & 1.82 & $0.20-18.32$ & 0.427 \\
\hline
\end{tabular}

LRP1B Low density lipoprotein receptor-related protein 1B, Col28A1 Collagen type XXVIII alpha 1 chain, FAM180A Family with sequence similarity 180 , member $\mathrm{A}$, PERP2 TP53 apoptosis effector, CHST1 Carbohydrate sulfotransferase 1 and PLIN1 Perilipin-1, FDR (False Discovery Rate), LogFC (Log Fold Change)

(Carbohydrate Sulfotransferase 1) is a membrane bound sulfotransferase potentially involved in the production of L-selectin ligands, important for the recognition of various immunological receptors involved with the recruitment of macrophages to the tissue inflammation sites [30]. In addition, the GAGs can bind to many genes responsible for ossification and osteoblast formation, such as fibroblast growth factors (FGFs) and bone morphogenetic proteins (BMPs), regulating the skeletogenesis [31]. CHST1 differential expression pattern between health and FHS-affected group was also confirmed by qPCR (Table 2). Moreover, using the same experimental population, Petry et al. [32] found that Adiponectin (ADIPOQ), Paired related Homeobox 1 (PRRX1), ABI family member 3 NESH binding protein $P(A B I 3 B)$ and COL8A1 genes were also downregulated in FHS-affected group by $\mathrm{qPCR}$, confirming their involvement with ECM, cell adhesion and bone formation.
According to the expression profile in this casecontrol study, a large number of coding genes for ECM structural proteins were downregulated, such as FN1 (Fibronectin 1), FBLN7 (fibulin 7), DPT (dermatopontin), GPC4 (glypican 4), BCAN (brevican), FMOD (fibromodulin), FBN2 (fibrillin 2), VIT (vitrin), CHPF (chondroitin polymerizing factor), SDK2 (sidekick cell adhesion molecule 2), DBN1 (drebrin 1), SDC3 (syndecan 3), ITGA11 (integrin, alpha 11) and OGN (osteoglycin) (Fig. 5). Also, several members of the collagen family were downregulated in FHS-affected broilers: COL6A1 (collagen, type VI, alpha 1), COL6A2 (collagen, type VI, alpha 2), COL6A3 (collagen, type VI, alpha 3 ), COL8A1 (collagen, type VIII, alpha 1), COL14A1 (collagen, type XIV, alpha 1), COL18A1 (collagen type XVIII alpha 1 chain) and COL28A1 (collagen, type XXVIII, alpha 1). Here, the FN1 gene can be highlighted, because it is a multidomain glycoprotein with multiple adhesive

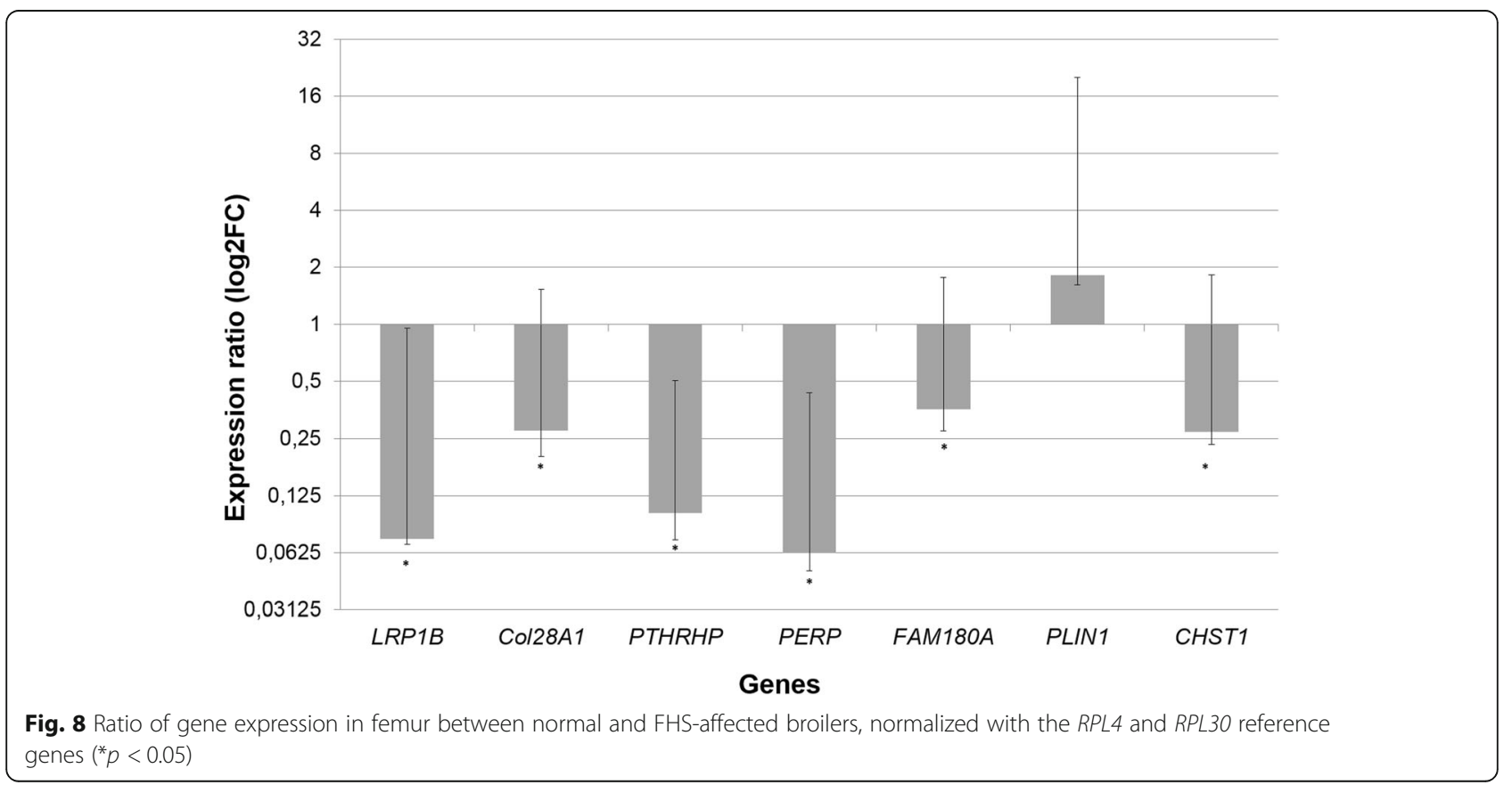


properties binding cells and their extracellular matrices, and was recognized to be the target for a large number of bacteria [33]. The FN1, Col8A1, Col18A1 and MFGE8 are also involved with angiogenesis (Fig. 7), and both FN1 and Col18A1 were downregulated in FHS-affected broilers, interacting with other BP (Fig. 6).

Moreover, most of the $\mathrm{DE}$ genes in our study presented binding molecular functions (MF), such as binding to ions, protein, heterocyclic compound and organic cyclic compound (Fig. 3). The molecular binding function is tightly coupled with the pathological condition evaluated in this study, since FHS is the separation of the growth plate from the articular cartilage [14]. The binding function is an intrinsic ability of the ECM macromolecule components and it is essential to the cell-matrix interactions. Several BP of cell adhesion related to binding MF were enriched in our study: biological adhesion, cell adhesion, regulation of cell adhesion, cell-matrix adhesion, cell-substrate adhesion, cell adhesion mediated by integrin, positive regulation of cell adhesion, cell-cell adhesion, single organismal cellcell adhesion and single organism cell adhesion (Additional file 3, Fig. 7). Most of the genes grouped in these previous BP were downregulated in the FHS-affected broilers, indicating reduction in cell adhesion and binding function in the femur GP. Altogether, the results indicate that the downregulation of the ECM genes could negatively affect the ECM integrity predisposing to the separation of growth plate from articular cartilage in the femur epiphysis. Since cell adhesion and ECM related genes are connecting the main $\mathrm{BP}$ identified in this study, it is possible that the disruption in these processes could be the first steps to initiate FHS in chickens.

\section{Blood circulation and angiogenesis}

Another observed supercluster in REViGO analysis (Fig. 4) is blood circulation that grouped important BP related to bone development: blood circulation, hemopoiesis, angiogenesis and circulatory system development (Additional file 3). Blood circulation is connected with other observed BP, such as immune response and inflammation. It is known that tissue damage leads to an increase in blood flow, capillary permeability and in the influx of immune cells to repair the tissue. In BCO lesion, affected blood vessels may be completely occluded by bacteria or may contain bacterial clumps surrounded by inflammatory cells and fibrin thrombi [16]. This group of blood circulation BP was enriched with upregulated genes, such as $A D A, A G T, C B S, C E B P B, E P B 42$, GFI1B, IL7R, IRF1, JAK2, NPR3, PDGFRA, RSAD2 and RHAG, and downregulated genes in the affected group, such as ADIPOQ, COL8A1, FN1, GLI2, HSPB1, LOXL2, MEOX2, MFAP5, MYH15, PTCH1, PRRX1, SFRP1, SREBF1, THBS1 and TNMD (Fig. 6, Fig. 7). An interesting gene grouped in this $\mathrm{BP}$ is $L O X L 2$, which has biological functions in the regulation of angiogenesis [34], being intrinsically related to the hypoxic state [35]. The bone development is tightly connected to the normal growth of blood vessels during endochondral formation [36, 37] and the vascularization is essential for physiological processes of the skeletal system. Blood vessels control bone growth and homeostasis by angiogenic and angiocrine signals mediating the transport of circulating cells, oxygen, nutrients and waste products [36]. Low levels of LOXL2 gene, a member of hypoxia-induced LOX, could contribute to the lack of vascularization in the growth plate of the femur. This pattern has already been observed in studies with human and mice $[35,38]$. This gene is also essential to the ECM formation, as described previously. Therefore, angiogenesis and vascularization seem to be secondary processes involved in FHS.

Angiopoietin 4 (ANGPT4) was upregulated in broilers with FHS. The angiopoietins are proteins with important role in vascular development and angiogenesis. However, this gene was not grouped in any BP using DAVID. In this study, Angiopoietin-like 5 (ANGPTL5) was one of the top 10 downregulated genes (Table 1) and Petry et al. [32] have confirmed the downregulation of ANGPTL5 in FHS-affected broilers by qPCR. Angiopoietin-like proteins (ANGPTLs) are a family of proteins structurally similar to the angiopoietins, with functional roles in lipid metabolism, glucose metabolism, inflammation and hematopoiesis [39].

\section{Lipid metabolism}

Some DE genes identified in our study were involved with lipid biological processes, such as: neutral lipid metabolic process, triglyceride metabolic process, lipid transport, lipid localization, regulation of lipid transport, cholesterol transport, fat cell differentiation and brown fat cell differentiation. The ABCA8, ALDH6A1, APOA1, CEBPB, OSBPL1A, P2RX7, PNPLA2, AGT and PDK4 genes were upregulated in affected broilers while SREBF1, SFRP1, PTCH1, THBS1 and ADIPOQ were downregulated (Additional file 2 and Additional file 3). Durairaj et al. [15] consider FHS as a metabolic skeletal problem in fast-growing chickens and observed elevation in blood lipid levels in broilers with FHS, suggesting that elevated lipid levels may be a risk factor for femoral head problems in chickens. Here, the ADIPOQ was downregulated in the femur of FHS-affected broilers and its expression profile was confirmed by qPCR [32]. According to DAVID results, $A D I P O Q$ participates in 107 out of 226 BP mapped in this case-control study (Additional file 3), including bone development, cell adhesion, immune response and apoptosis, except homeostasis. In broilers, $A D I P O Q$ acts in the metabolism of lipids and carbohydrates, being involved in adipogenesis [40]. Moreover, 
adiponectin has important role in regulating bone metabolism, inhibiting the osteoprotegerin expression in the osteoblasts and, also, by increasing the differentiation of osteoclasts [41]. Therefore, the $A D I P O Q$ is an important candidate gene, since it has already been associated with metabolic disorders, including arthritis, osteonecrosis and obesity in humans [42-44]. Moreover, this gene acts in several BP possibly involved with the causes of FHS in broilers, as discussed in our study, including lipidemia.

Furthermore, adiponectin binds to specific receptors, ADIPOR1 and ADIPOR2. The ADIPOR1 is the major receptor that mediates the glucose and lipid metabolism-related effects of adiponectin on target cells [45]. Adiponectin gene may be associated with adipose tissue deposition in chickens [40, 46]. Cruz et al. [47] found a SNP ( $729 \mathrm{C}>\mathrm{T}$ ) in the ADIPOR1 gene associated with thickness of the femur, suggesting its influence on bone integrity in broilers.

An important candidate gene to FHS condition in broilers is the purinergic receptor P2X7 (P2RX7), a member of the purinoceptors for ATP family [48]. This gene participated in $102 \mathrm{BP}$ in DAVID analyses (Additional file 3) and was involved in the majority of the BP discussed in this study. Besides being essential in DNA and RNA synthesis, purines are important components of several biomolecules associated to the energetic metabolism such as ATP, GTP, cAMP and NADH. In this study, two purine BP were found: purine nucleotide salvage, including genes $A M P D 3$ and $A D A$, and purinecontaining compound salvage, including genes CECR1, $A M P D 3$ and $A D A$. Here, adenosine monophosphate deaminase 3 (AMPD3) and adenosine deaminase (ADA) were upregulated in FHS-affected broilers and the CECR1, also named adenosine deaminase (ADA2), was downregulated. Also included in the purinergic metabolism is the CHAT2 gene, the most upregulated gene in the FHS-affected broiler (Table 1).

In this transcriptome study, the G0/G1 switch gene 2 (GOS2), a candidate gene for metabolic disorders, was upregulated in broilers with FHS. This gene has been considered a potent endogenous inhibitor of adipose triglyceride lipase (ATGL) revealing a unique mechanism governing lipolysis and fatty acid availability [49]. Zhang and coworkers [44] evidenced that the GOS2 acts as a master regulator of tissue-specific balance of triglycerides storage, mobilization, partitioning of metabolic fuels between adipose and liver, and of the whole-body adaptive energy response.

About $64 \%$ of the genes grouped in BP related to lipid transportation and metabolism were upregulated, such as APOA1, PNPLA2, ABCA8, P2RX7, OSBPL1A, $A L D H 6 A 1, C E B P B, A G T$ and PDK4. Some of them also interact with genes involved in the ossification and inflammation (Fig. 5, Fig. 6). The lipids play a role in bone metabolism, since they can affect the function of bone cells through many pathways [50], such as the vitamin D and $\mathrm{K}$ absorption, regulating the mineral maturation and also affecting the bone mineral density [50]. Those lipids can be driven to the bone via circulation, but could also be produced by osteoblasts [51]. Therefore, accumulation of lipids in the bone cells might lead to an inflammatory environment, generating oxidative stress, which could inhibit the expression of bone differentiation markers [52]. Thus, the unbalance of the lipid metabolism could precede the cell adhesion and ECM bioprocesses on the onset of FHS in fast-growing broilers.

\section{Immune response genes}

Immune biological processes were overrepresented in both Blast2GO and REViGO. The profile of DE genes observed in this study showed a global activation of the immune system, comprehending innate immunity genes and genes associated with the inflammatory response. Some of the main BP found in DAVID were: immune system process, immune system development, regulation of immune system process, $\mathrm{T}$ cell mediated immunity, leukocyte differentiation, immune response, response to cytokine, leukocyte activation, leukocyte proliferation, lymphocyte activation, inflammatory response, defense response, regulation of defense response, defense response to other organism and defense response to bacterium (Additional file 3). Among the enriched genes, $A D A, A L O X 5 A P, A M P D 3, A P O A 1, A V D, C 7, C E B P B$, EPB42, FYB, GFI1B, IL7R, IRF1, JAK2, NLRC5, NPR3, PTPRC, PDK4, P2RX7, PDGFRA, RHAG and RSAD2 were upregulated in the epiphysiolysis group, while $A D I$ POQ, CD151, CHGA, FN1, HSPB1, MFAP5, SFRP1, TINAGL1 and THBS1 were downregulated. The upregulated genes were predominant in all 20 immune BP.

Several response BP were observed, including defense response to other organism enriched with genes NLRC5, P2RX7, CHGA, CEBPB, AVD, PDK4, IRF1 and RSAD2, and defense response to bacterium enriched with P2RX7, CHGA, CEBPB and AVD genes. In both BP, only CHGA was downregulated in affected chickens. Although FHS may precede bacterial infection, these two $\mathrm{BP}$ are indicative of the presence of microorganisms in the GP tissue. The P2RX7 receptor serves as a pattern recognition receptor for extracellular ATP-mediated apoptotic cell death [53] and inflammation [54]. P2RX7 mediates NLRP3 inflammasome activation, cytokine and chemokine release, $\mathrm{T}$ lymphocyte survival and differentiation, transcription factor activation, and cell death [55]. In humans, Saunders et al. [48] found that a SNP in the $P 2 R X 7$ gene failed to induce apoptosis and abolishes the P2X7-mediated pathway allowing survival of mycobacteria within infected host cells. 
The avidin $(A V D)$, another interesting gene involved in the defense response to bacterium $\mathrm{BP}$, was one the most upregulated in the femur of affected broilers, suggesting a potential role in the resilience of chickens. The $A V D$ is a biotin-binding glycoprotein expressed under inflammation in several chicken tissues [56], acting as a defense protein protecting the development of the chicken embryo against microbial infections [57]. Packialakshmi et al. [58] found elevated levels of Gallinacin-9 (GAL9), another chicken defense peptide, in plasma of FHN-affected 35 day-old commercial broilers compared to normal broilers.

The cytokines are a family of secreted proteins involved in immunoregulatory and inflammatory processes. In our study, C-C motif chemokine ligand 19 (CCL19) was one of the most expressed genes in the affected chickens (Table 1). The upregulation of CCL19 and CCL21 and their receptor CCR7 was associated to Rheumatoid arthritis pathogenesis, playing an important role in bone destruction by increasing osteoclast migration and resorption activity [59]. Therefore, genes involved in inflammation could lead to a disruption in the bone development of fast-growing chickens and be another possible cause of FHS.

The increased levels of antimicrobial peptide genes in the growth plate of FHS-affected broilers found in our study could indicate microbial infection of the femur. Femur epiphysiolysis has been implicated in subsequent problems, such as bacterial infection [6]. The femoral head damage compromises the physiological barrier against infections and can lead to FHN and osteomyelitis [15], conditions potentially related to $\mathrm{BCO}$. The $\mathrm{BCO}$ could be initiated by mechanical microfracturing of the growth plate, followed by colonization of osteochondrotic clefts by a wide range of different opportunistic bacteria hematogenously dispersed [60]. The most frequent organism associated to osteomyelitis is Staphylococcus aureus [61, 62]. The ability of $S$. aureus to infect the osteoblast is highly correlated to its ability to bind to the extracellular matrix, and multiple adhesins produced by the bacteria have strong interaction with components of the avian extracellular matrix [63-65]. After osteoblasts infection, bacterium modulates the production of cytokines and chemokines, together with the increase of osteoclastogenesis and death of osteoblasts, increasing the imbalance of bone homeostasis $[64,65]$. Therefore, besides its structural function, the ECM also modulates the microorganism colonization in the growth plate tissue. The immune response BP grouped genes possibly involved with the cause and consequence of the FHS. Those related to defense response to other organism and response to bacteria seem to be a consequence of FHS, while genes enriching inflammatory BP could be triggering this disorder in commercial broilers.

\section{Apoptosis or cell death}

Cell death is a physiological mechanism probably involved in the FHS in broilers due to its essential role in development and tissue homeostasis. The forms of cell death (necrosis, apoptosis and autophagy) are not separated by strict boundaries sharing molecular effectors and signaling ways [66]. In our study, biological process associated to cell death, such as regulation of cell death, regulation of apoptotic process, programmed cell death and regulation of programmed cell death (Additional file 3) grouped $A D A, A G T, C E B P B, G O S 2$, IRF1, JAK2, OSGIN1, P2RX7 and PDK4 genes that were upregulated in FHS-affected broilers, while $A D I P O Q$, AIFM2, CRIP1, CTSD, GLI2, HSPB1, SFRP1 and THBS1 were downregulated.

The P2RX7 gene can be highlighted because its activation stimulates downstream events, triggering the caspase cascade and leading to apoptotic death of the target cell [67]. Another interesting gene related to apoptosis is the TP53 apoptosis effector (PERP2), which was not recognized in BP with DAVID database. According to KEGG p53 signaling pathway, the p53 activation is induced by a number of stress signals, including DNA damage, oxidative stress and activated oncogenes. The p53 protein is employed as a transcriptional activator of p53-regulated genes resulting in cell cycle arrest, cellular senescence or apoptosis. In our study, PERP2 was one of the most downregulated genes in the GP transcriptome, being 3.04 times less expressed in the affected than in the normal group. This expression profile was confirmed by the qPCR analysis, with PERP2 being 16.1 times less expressed in FHS-affected than in normal broilers (Table 2 ). In chickens, the function of PERP2 has not been widely studied. However, this gene has already been associated with epithelial diseases in humans, mainly because of its function on epithelial integrity and cell-cell adhesion, due to an impaired intercellular adhesion observed after mechanical stress [68]. This finding is interesting since one of the possible causes of FHS and FHN in broilers is due to mechanical stress triggered by the rapid growth and heavy weight of modern broilers.

The GOS2 gene was upregulated and was enriched in the apoptosis and cell death pathways (Additional file 3). GOS2 promotes apoptosis by interacting with the antiapoptotic protein $\mathrm{Bcl}-2$, and downregulation of GOS2 expression could be a prime avenue for growing cells, especially cancer cells, in preventing death or growth arrest. Despite the GOS2 pro-apoptotic function, this mechanism may be cell type-specific. The presence of high levels of GOS2 does not seem to induce apoptosis in adipocytes, which are known to be quite resilient to apoptotic death [69].

Even though FHS is commonly observed in meat-type chicken, in this case and control study, response to 
stress, cell death and immune response processes were $\mathrm{BP}$ highly represented. The high number of $\mathrm{DE}$ genes in these processes gives insight on the subclinical implications of epiphysiolysis in broilers. The disruption of these biological processes may impact femur head development and lead to more severe femur disorders, such as necrosis and osteomyelitis.

This study identified several pathways and biological processes related to FHS, corroborating with the major hypotheses regarding the causes of this disorder. Furthermore, we highlighted that the impaired cell adhesion and ECM seem to be the most important processes to trigger FHS in chickens. These mechanisms have not been widely explored if compared to those related to angiogenesis and bone development. Moreover, DE genes related to inflammation and lipidemia were also enriched in the BP found in our study. The role of these mechanisms in metabolic disorders, including those related to bone, has recently been discussed, especially in humans. It is known that inflammatory diseases are associated to bone losses, bone resorption and obesity [70,71]. Therefore, the fast growth of broilers, mainly around 35 days of age, could increase the expression of inflammatory and lipid related genes, influencing the development of FHS in broilers. These findings are in agreement with studies that did not find any bone quality issue in fast-growing chickens evaluated in early ages of development.

Understanding the changes in the GP transcriptome might support breeding strategies to obtain methods to address poultry robustness and more resilient broilers. Furthermore, identifying young broilers with vulnerable femoral joint can help genetic selection against FHS. Currently, clinical signs of FHN are not detectable at early stages. The diagnoses of these anomalies are done at late stage due to lameness or after necropsy. Noninvasive biomarkers that can help genetic selection of broilers against FHS are required to assess the predisposition to FHN [13]. Thus, some of the proteins coded by $\mathrm{DE}$ genes such as LOXL2, ADA, PERP2, ADIPOQ, $M A P K 12, F N 1, P 2 R X 7$ and $A V D$ found in our study should be investigated as biomarkers, which could be easily evaluated in the blood. Moreover, further functional analyses of these genes are interesting to elucidate their contribution to the development of the femur head necrosis in chickens, which would also be important for the understanding of humans bone disorders.

\section{Conclusions}

Genes with potential role in epiphysiolysis were identified through the femur head transcriptome analysis, providing a better understanding of the mechanisms that regulate bone development in fast-growing chickens. In this study, we highlighted the importance of cell adhesion and extracellular matrix related genes in triggering
FHS. The imbalance in expression of the genes involved in these processes could make broilers more susceptible to this condition. Furthermore, we have shown that, besides the angiogenesis and bone development genes expected to be DE in FHS, new findings were described associating lipidemia and immune response/inflammation with FHS in broilers.

\section{Methods}

\section{Experimental animals and sample collection}

This study was performed with the approval of the Embrapa Swine and Poultry Ethical Committee of Animal Use (CEUA) under protocol number 012/2012. Commercial male broilers (Cobb500) with 35 days of age from a poultry farm located in Concórdia, Santa Catarina State, Brazil, were used. The chickens were housed according to optimal industry growing standard practices for this line and raised with free access to both feed and water. To reduce environmental effects, broilers used in this study were sampled from the same flock, in a Darkhouse system managed by a high standard producer. Based on visual observation, a total of 29 individuals, including 14 healthy and 15 lameness-affected chickens, were sampled and transferred to the Embrapa Swine and Poultry National Research Center, located in ConcordiaSC, for tissue sample collection. The body score was evaluated in all animals prior to necropsy. Broilers were euthanized by cervical dislocation followed by bleeding.

The proximal femoral head was classified according to the presence or absence of different levels of $\mathrm{BCO}$, based upon clinical examination of the growth plate (GP) separation from the articular cartilage (AC) by visual observation of compatible necrosis lesions, according to Wideman et al. [12]. Femoral samples with good adhesion between GP and AC were considered in the normal group. On the other hand, samples presenting separation between GP and AC showing no lesions in GP comprised the proximal femoral head separation (FHS) or epiphysiolysis group. In the proximal femoral head region from all broilers, samples were collected from GP, stored in liquid nitrogen and transferred to the $-80^{\circ} \mathrm{C}$ freezer.

\section{RNA isolation, CDNA library and sequencing}

Total RNA was isolated from $100 \mathrm{mg}$ of the femoral head tissue using Trizol Reagent (Invitrogen, Carlsbad, CA) following the manufacturer's instructions. The RNA cleanup was performed using RNeasy mini kit (Qiagen, Germany) also following the manufacturer's instructions. Total RNA was quantified in Nanodrop spectrophotometer (Thermo Scientific; Waltham, MA, USA) and the integrity was assessed using an Agilent 2100 BioAnalyzer (Agilent Technologies; Santa Clara, CA, USA). Samples with RNA integrity number (RIN) higher than 8 were considered for 
further analysis. A total of eight samples of GP, four normal and four FHS-affected were prepared for RNAsequencing. Barcoded Illumina sequencing libraries for gene expression profile were obtained for each of the 8 individuals using the TruSeq ${ }^{\mathrm{ma}}$ RNA Sample Prep Kit v2 (Illumina, Inc.; San Diego CA, USA), according to the manufacturer's recommendations, with $2 \mu \mathrm{g}$ of total RNA. Libraries were submitted to the Functional Genomics Center, ESALQ, University of São Paulo, Piracicaba, São Paulo State, Brazil for sequencing in Illumina HiSeq2500 equipment (Illumina, Inc.; San Diego CA, EUA), all in the same lane, following the 2x100bp paired-end protocol.

\section{Transcriptome analyses}

Raw FASTQ reads were submitted to quality control using the SeqyClean tool [72] for removing short reads (<70 bp), low quality reads (QPhred $<24$ ) and adapter sequences. Sequence reads were mapped to the chicken reference genome (Gallus gallus, assembly 5.0) available in Ensembl 87 (www.ensembl.org) using the BWA-MEM software [73]. The counts of fragments were obtained using the HTseq software [74]. The differentially expressed (DE) genes were identified using the edgeR package [75]. The DE genes between FHS-affected and healthy broilers were selected based on the level of False Discovery Rate $(\mathrm{FDR} \leq 0.05)$ after the BenjaminiHochberg (BH) method for multiple correction tests [76]. To verify the expression pattern between FHSaffected and unaffected groups, the LogFC values from each gene were used to create multidimensional scaling $(M D S)$ plots using R. Based on DE genes, a heatmap was generated to check the consistence between samples using R. The FASTQ files obtained in this study were deposited in the SRA database, with BioProject number PRJNA352962.

\section{Functional analyses}

The functional annotation analyses were performed based on the DE genes with FDR $<0.05$. The FASTA files of these genes obtained in the Ensembl 87 were submitted to the Blast2GO software [77] for the gene ontology (GO) search. Furthermore, the DAVID database (http://david.abcc.ncifcrf.gov/tools.jsp) was used to improve the biological processes (BP) obtained in the Blast2GO software, where the expressed genes in this study were set as background. Once obtained the BP, the GO numbers from DAVID were clustered by the REViGO tool [78] for easily visualization and understanding. Genes with positive LogFC values were considered upregulated while those with negative values were downregulated in the FHS-affected broilers compared to the normal group. Gene networks were constructed using Stringdb and the NetworkAnalyst tools [79, 80].
Table 3 Primers of the target and normalizing genes used in the quantitative PCR analyses

\begin{tabular}{lll}
\hline Gene & Primers sequence $\left(5^{\prime}-\right.$ 3') $^{\prime}$ & Ensembl ID \\
\hline LRP1B & F: GGCTACTGCTACAATGGTGGT & ENSGALG00000012407 \\
& R: GTTGGCGGAGCATAGACAGA & \\
COL28A1 & F: ACCAGGTCTAAAGGGTCAACG & ENSGALG00000010259 \\
& R: TGGATTCCCAGAGTCTCCCA & \\
PERP2 & F: TGGACTATGGATGGGGGAGAG & ENSGALG00000027207 \\
& R: GAGGGCGAACAGATGACCA & \\
FAM180A & F:GAGTAGAGCTATGCTTACCCAGC & ENSGALG00000028459 \\
& R: CGAAGCCAGCTCCTCATCTT & \\
PLIN1 & F: GGCTATGGAGACGGTGGATG & ENSGALG00000023395 \\
& R: CTGGCTTGCTCTCCTCTTCC & \\
CHST1 & F: CGCCCCTCTTTCTCGTCTTC & ENSGALG00000008440 \\
& R: GCTTGGAGAGACCCGATCC & \\
RPL4* & F: TGTITGCCCCAACCAAGACT & ENSGALG00000007711 \\
& R: CTCCTCAATGCGGTGACCTT & \\
RPL30 ${ }^{a}$ & F: ATGATTCGGCAAGGCAAAGC & ENSGALG00000008212 \\
& R: GTCAGAGTCACCTGGGTCAA & \\
&
\end{tabular}

${ }^{a}$ Petry et al. [32]

\section{Validation by quantitative PCR (qPCR)}

Quantitative PCR analysis was used to confirm the expression pattern of six DE genes in the GP transcriptome using 20 samples of 35 day-old broilers (10 normal and 10 affected). The genes selected were: Low density lipoprotein receptor-related protein $1 B$ (LRP1B), Collagen type XXVIII alpha 1 chain (Col28A1), Family with sequence similarity 180, member A (FAM180A), TP53 apoptosis effector (PERP2), Carbohydrate sulfotransferase 1 (CHST1) and Perilipin-1 (PLIN1). Those genes were selected based on their LogFC, FDR and function. Sequences were obtained from Gallus gallus on Genebank (http://www.ncbi.nlm.nih.gov/gene/) and Ensembl (www. ensembl.org). Primers used for each gene (Table 3) were designed in exon-exon junctions using the Primer-Blast online tool [81]. The primers' quality was evaluated in the NetPrimer online software (http://www.premierbio soft.com/netprimer/). The Ribosomal protein 4 (RPL4) and Ribosomal protein 30 (RPL30) genes were used as reference [32]. The qPCR reactions were carried out in QuantStudio 6 (Applied Biosystems, USA) equipment, in a final volume of $15 \mu \mathrm{L}$ containing $1 \mathrm{X}$ Maxima Mastermix SYBR Green (Fermentas, USA), $0.133 \mu \mathrm{M}$ for each primer and $2 \mu \mathrm{L}$ of cDNA. The cycle threshold $(\mathrm{Ct})$ mean for each replicate sample was obtained and normalized with the reference genes. The relative quantification analysis was performed using the Relative Expression Software Tool (REST, [82]) that applies a pair wise fixed reallocation randomization test, a nonparametric statistical test. 


\section{Supplementary information}

Supplementary information accompanies this paper at https://doi.org/10. 1186/s12864-019-6411-9.

Additional file 1. Multi-Dimensional Scaling Plot (MDS-plot) measuring the similarity of the samples into 2-dimensions (blue represents the normal group and red the affected group).

Additional file 2. An excel file with the expressed and DE genes between normal and FHS-affected broilers with 35 days of age.

Additional file 3. $\mathrm{GO}$ biological process enriched based on the differentially expressed genes between normal and FHS-affected broilers.

\section{Abbreviations}

AC: Articular cartilage; ATP: Adenosine triphosphate; BCO: Bacterial Chondronecrosis with Osteomyelitis; BP: Biological processes; CAMP: Adenosine 3',5'monophosphate; CEUA: Ethical Committee of Animal Use; Ct: Cycle threshold; DE: Differentially expressed; ECM: Extracellular matrix; FDR: False Discovery Rate; FGFs: Fibroblast growth factors: FHN: Femoral Head Necrosis; FHS: Femoral head separation; GAGs: Glycosaminoglycans; GP: Growth plate; LogFC: Log fold change; MDS: Multi-dimensional scaling; MF: Molecular functions; qPCR: Quantitative $P C R$

\section{Acknowledgements}

The authors are grateful to Alexandre L. Tessmann and the Embrapa Bioinformatics Multi-Users Laboratory from Embrapa Informática Agropecuária for technical assistance. JAPM and IRS were a PIBIC/CNPq fellow at Embrapa Swine and Poultry National Research Center. RZ was supported by a BJT/CNPq grant n³73167/2012. MLS was supported by a PVE/CNPq grant n 407404/2013-9. MCL and LLC are CNPq fellows.

\section{Authors' contributions}

AMGI, JOP and MCL conceived and designed the experiment. JOP, MCL, RZ, JRP and FRFJ were responsible for the data collection. MEC, AMGI and MLS performed the RNA-Seq analysis. AMGI, AZ and PFG performed the functional analyses of the genes. IRS performed validation analysis. AMGI, JAPM, JOP, LLC and MCL interpreted the results and evaluated the conclusions. JOP wrote the manuscript. All authors reviewed, edited and approved the final manuscript.

\section{Funding}

This study was funded by the Brazilian Agricultural Research Corporation (Embrapa), project number 01.11.07.002.04.03, from the Brazilian Government.

\section{Availability of data and materials}

The datasets used or analyzed during the current study are available from the corresponding author on reasonable request. The transcriptome sequences are available in the SRA database with BioProject number PRJNA352962 and biosample numbers: SAMN06007242, SAMN06007241, SAMN06007240, SAMN06007239, SAMN06007238, SAMN06007237, SAMN06007236 and SAMN06007235.

\section{Ethics approval}

All animal procedures were performed in accordance with the Ethics Committee on Animal Utilization of the Embrapa Swine and Poultry National Research Center under protocol number 012/2012.

\section{Consent for publication}

Not applicable.

\section{Competing interests}

The authors declare that they have no competing interests.

\section{Author details}

'Embrapa Suínos e Aves, Rodovia BR-153, Km 110, Distrito de Tamanduá, Caixa Postal: 321, Concórdia, Santa Catarina 89715-899, Brazil. ${ }^{2}$ Programa de Pós-Graduação em Ciências Veterinárias, Universidade Estadual do Centro-Oeste, Guarapuava, Paraná, Brazil. ${ }^{3}$ Universidade do Contestado,
Concórdia, Santa Catarina, Brazil. ${ }^{4}$ Programa de Pós-Graduação em Zootecnia, UDESC-Oeste, Chapecó, SC, Brazil. ${ }^{5}$ Embrapa Informática Agropecuária, Campinas, SP, Brazil. ${ }^{6}$ UC Davis Genome Center, University of California, Davis, CA, USA. ${ }^{7}$ Universidade de Passo Fundo, Passo Fundo, RS, Brazil.

${ }^{8}$ Programa de Mestrado em BioExperimentação, UPF, Passo Fundo, RS, Brazil. ${ }^{9}$ Department of Genetics, Ribeirão Preto Medical School, University of São Paulo, Ribeirão Preto, SP, Brazil. ${ }^{10}$ Universidade de São Paulo, Piracicaba, SP Brazil.

Received: 14 November 2018 Accepted: 18 December 2019

Published online: 30 December 2019

\section{References}

1. FAO. Sources of Meat. 2014

2. Havenstein GB, Ferket PR, Qureshi MA. Growth, livability, and feed conversion of 1957 versus 2001 broilers when fed representative 1957 and 2001 broiler diets. Poult Sci. 2003.

3. Zuidhof MJ, Schneider BL, Carney VL, Korver DR, Robinson FE. Growth, efficiency, and yield of commercial broilers from 1957, 1978, and 2005. Poult Sci. 2014:93(12):2970-82

4. Gerbens-Leenes PW, Mekonnen MM, Hoekstra AY. The water footprint of poultry, pork and beef: a comparative study in different countries and production systems. Water Resour Ind. 2013;1-2:25-36.

5. Tallentire CW, Leinonen I, Kyriazakis I. Breeding for efficiency in the broiler chicken: a review. Agron Sustain Dev. 2016.

6. Julian RJ. Production and growth related disorders and other metabolic diseases of poultry - a review. Vet J. 2005;169(3):350-69.

7. Bradshaw RH, Kirkden RD, Broom DM. A review of the aetiology and pathology of leg weakness in broilers in relation to welfare. Avian Poult Biol Rev. 2002;13(2):45-103.

8. de Jong I, Berg C, Butterworth A, Estevéz I. Scientific report updating the EFSA opinions on the welfare of broilers and broiler breeders. Eur Food Saf Auth. 2012:EN-295. https://doi.org/10.2903/sp.efsa.2012.EN-295.

9. FAWC. Five Freedoms. Farm Animal Welfare Council. 2009. https://www.gov uk/government/publications/fawc-report-on-farm-animal-welfare-in-greatbritain-past-present-and-future. Accessed 20 June 2019

10. Singh Sanotra G, Berg C, Damkjer Lund J. A comparison between leg problem in Danish and Swedish broiler production. Anim Welf. 2003;12(4): $677-83$

11. Knowles TG, Kestin SC, Haslam SM, Brown SN, Green LE, Butterworth A, et al. Leg disorders in broiler chickens: prevalence, risk factors and prevention. PLoS One. 2008;3(2):e1545.

12. Wideman RF, Hamal KR, Stark JM, Blankenship J, Lester H, Mitchell KN, et al. A wire-flooring model 1 for inducing lameness in broilers: evaluation of probiotics as a prophylactic treatment. Poult Sci. 2012;91(4):870-83.

13. Packialakshmi B, Rath NC, Huff WE, Huff GR. Poultry femoral head separation and necrosis: a review. Avian Dis. 2015 Sep;59(3):349-54.

14. Riddell C, King MW, Gunasekera KR. Pathology of the skeleton and tendons of broiler chickens reared to roaster weights. Avian Dis: II. Normal chickens; 1983

15. Durairaj V, Okimoto R, Rasaputra K, Clark FD, Rath NC. Histopathology and serum clinical chemistry evaluation of broilers with femoral head separation disorder. Avian Dis. 2009;53(1):21-5.

16. McNamee PT, Smyth JA. Bacterial chondronecrosis with osteomyelitis ('femoral head necrosis') of broiler chickens: a review. Avian Pathology. 2000;29(4):253-70.

17. Dinev I. Clinical and morphological investigations on the prevalence of lameness associated with femoral head necrosis in broilers. Br Poult Sci. 2009;50(3):284-90.

18. Wideman RF, Prisby RD. Bone circulatory disturbances in the development of spontaneous bacterial chondronecrosis with osteomyelitis: A translational model for the pathogenesis of femoral head necrosis. Front Endocrinol (Lausanne). 2013:3(JAN):1-14

19. Van Der Eerden BCJ, Karperien M, Wit JM. Systemic and Local Regulation of the Growth Plate. Endocrine Reviews. 2003;24(6):782-801.

20. Johnsson M, Jonsson KB, Andersson L, Jensen P, Wright D. Genetic regulation of bone metabolism in the chicken: similarities and differences to mammalian systems. PLoS Genet. 2015;11(5):e1005250.

21. Applegate TJ, Lilburn MS. Growth of the femur and tibia of a commercial broiler line. Poult Sci. 2002;81(9):1289-94. 
22. Williams B, Solomon S, Waddington D, Thorp B, Farquharson G. Skeletal development in the meat-type chicken. Br Poult Sci. 2000;41(2):141-9.

23. Yalcin S, Özkan S, Coşkuner E, Bilgen G, Delen Y, Kurtulmuş Y, et al. Effects of strain, maternal age and sex on morphological characteristics and composition of tibial bone in broilers. Br Poult Sci. 2001;42(2):184-90.

24. Yair R, Uni Z, Shahar R. Bone characteristics of late-term embryonic and hatchling broilers: bone development under extreme growth rate. Poult Sci. 2012;91(10):2614-20.

25. Kestin SC, Gordon S, Su G, Sørensen P. Relationships in broiler chickens between lameness, liveweight, growth rate and age. Vet Rec. 2001;148: 195-197.

26. Damaziak K, Charuta A, Niemiec J, Tatara MR, Krupski W, Gozdowski D, et al. Femur and tibia development in meat-type chickens with different growth potential for 56 days of rearing period. Poult Sci. 2019;98(12):7063-75.

27. Velleman SG. The role of the extracellular matrix in skeletal muscle development. Poult Sci. 2000;79:985-9.

28. Lopez-Jimenez AJ, Basak T, Vanacore RM. Proteolytic processing of Lysyl oxidase like- 2 in the extracellular matrix is required for crosslinking of basement membrane collagen IV. J Biol Chem. 2017;292(41):16970-82

29. Lucero HA, Kagan HM. Lysyl oxidase: an oxidative enzyme and effector of cell function. Cell Mol Life Sci. 2006;63(19-20):2304-16.

30. Li X, Tu L, Murphy PG, Kadono T, Steeber DA, Tedder TF. CHST1 and CHST2 sulfotransferase expression by vascular endothelial cells regulates shearresistant leukocyte rolling via L-selectin. J Leukoc Biol. 2001;69(4):565-74.

31. Benoit DSW, Anseth KS. Heparin functionalized PEG gels that modulate protein adsorption for hMSC adhesion and differentiation. Acta Biomater. 2005;1(4):461-70.

32. Petry B, Savoldi IR, Ibelli AMG, Paludo E, de Oliveira Peixoto J, Jaenisch FRF, et al. New genes involved in the Bacterial Chondronecrosis with Osteomyelitis in commercial broilers. Livest Sci. 2018;208:33-9.

33. Henderson B, Nair S, Pallas J, Williams MA. Fibronectin: A multidomain host adhesin targeted by bacterial fibronectin-binding proteins. FEMS Microbiol Rev. 2011;35(1):147-200.

34. Bignon M, Pichol-Thievend C, Hardouin J, Malbouyres M, Bréchot N, Nasciutti $L$, et al. Lysyl oxidase-like protein-2 regulates sprouting angiogenesis and type IV collagen assembly in the endothelial basement membrane. Blood. 2011;118(14):3979-89.

35. Germain S, Monnot C, Muller L, Eichmann A. Hypoxia-driven angiogenesis: Role of tip cells and extracellular matrix scaffolding. Curr Opinion Hematol. 2010;17:245-51.

36. Huang SC, Zhang LH, Zhang JL, Rehman MU, Tong X Le, Qiu G, et al. Role and regulation of growth plate vascularization during coupling with osteogenesis in tibial dyschondroplasia of chickens. Sci Rep. 2018:8:3680 https://doi.org/10.1038/s41598-018-22109-y.

37. Ramasamy SK, Kusumbe AP, Schiller M, Zeuschner D, Bixel MG, Milia C, et al. Blood flow controls bone vascular function and osteogenesis. Nat Commun 2016;7:13601. https://doi.org/10.1038/ncomms13601.

38. Schietke R, Warnecke C, Wacker I, Schödel J, Mole DR, Campean V, et al. The lysyl oxidases LOX and LOXL2 are necessary and sufficient to repress Ecadherin in hypoxia: insights into cellular transformation processes mediated by HIF-1. J Biol Chem. 2010;285(9):6658-69.

39. Santulli G. Angiopoietin-like proteins: a comprehensive look. Front Endocrinol. 2014;5:4. https://doi.org/10.3389/fendo.2014.00004.

40. Tahmoorespur M, Ghazanfari S, Nobari K. Evaluation of adiponectin gene expression in the abdominal adipose tissue of broiler chickens: feed restriction, dietary energy, and protein influences adiponectin messenger ribonucleic acid expression. Poult Sci. 2010;89(10):2092-100.

41. Luo XH, Guo LJ, Xie H, Yuan LQ, Wu XP, Zhou H De, et al. Adiponectin stimulates RANKL and inhibits OPG expression in human osteoblasts through the MAPK signaling pathway. J Bone Miner Res. 2006;21(10): 1648-56.

42. Jiang L, Zhu X, Rong J, Xing B, Wang S, Liu A, et al. Obesity, osteoarthritis and genetic risk: the rs182052 polymorphism in the ADIPOQ gene is potentially associated with risk of knee osteoarthritis. Bone Joint Res. 2018;7(7):494-500.

43. Liu Z-J, Zhang H-W. Genetic polymorphisms of ADIPOQ and osteonecrosis of the femoral head risk in Chinese population. Int J Clin Exp Pathol. 2016; 9(8):7763-8

44. Kanu JS, Qiu S, Cheng Y, Li R, Kou C, Gu Y, et al. Associations between three common single nucleotide polymorphisms (rs266729, rs2241766, and rs1501299) of ADIPOQ and cardiovascular disease: a meta-analysis. Lipids Health Dis. 2018;17(1):126.
45. Jin Z, Pu L, Sun L, Chen W, Nan N, Li H, et al. Identification of susceptibility variants in ADIPOR1 gene associated with type 2 diabetes, coronary artery disease and the comorbidity of type 2 diabetes and coronary artery disease. PLoS One. 2014;9(6):e100339.

46. Maddineni S, Metzger S, Ocón O, Hendricks G, Ramachandran R. Adiponectin gene is expressed in multiple tissues in the chicken: food deprivation influences adiponectin messenger ribonucleic acid expression. Endocrinology. 2005;146(10):4250-6.

47. Da Cruz VAR, Schenkel FS, Savegnago RP, Grupioni NV, Stafuzza NB, Sargolzaei $\mathrm{M}$, et al. Association of apolipoprotein $\mathrm{B}$ and adiponectin receptor 1 genes with carcass, bone integrity and performance traits in a paternal broiler line. PLoS One. 2015;10(8):e0136824. https://doi.org/10.1371/journal.pone.0136824.

48. Saunders BM, Fernando SL, Sluyter R, Britton WJ, Wiley JS. A loss-of-function polymorphism in the human $\mathrm{P} 2 \mathrm{X} 7$ receptor abolishes ATP-mediated killing of mycobacteria. J Immunol. 2003;171(10):5442-6.

49. Zhang X, Heckmann BL, Campbell LE, Liu J. GOS2: a small giant controller of lipolysis and adipose-liver fatty acid flux. Biochim Biophys Acta Mol Cell Biol Lipids. 2017;1862(10 Pt B):1146-54.

50. Tintut $Y$, Demer LL. Effects of bioactive lipids and lipoproteins on bone. Trends Endocrinol Metabolism. 2014;25:53-9.

51. Brodeur MR, Brissette L, Falstrault L, Ouellet P, Moreau R. Influence of oxidized low-density lipoproteins (LDL) on the viability of osteoblastic cells. Free Radic Biol Med. 2008;44(4):506-17.

52. Parhami F, Jackson SM, Tintut $Y$, Le $V$, Balucan JP, Territo $M$, et al. Atherogenic diet and minimally oxidized low density lipoprotein inhibit osteogenic and promote adipogenic differentiation of marrow stromal cells. J Bone Miner Res. 1999;14(12):2067-78.

53. Kawano A, Tsukimoto M, Noguchi T, Hotta N, Harada H, Takenouchi T, et al. Involvement of P2X4 receptor in P2X7 receptor-dependent cell death of mouse macrophages. Biochem Biophys Res Commun. 2012;419(2):374-80.

54. Kurashima $\mathrm{Y}$, Kiyono $\mathrm{H}$. New era for mucosal mast cells: their roles in inflammation, allergic immune responses and adjuvant development. Experimental Molecular Med. 2014;46(3):e83. https://doi.org/10.1038/emm. 2014.7.

55. Di Virgilio F, Dal Ben D, Sarti AC, Giuliani AL, Falzoni S. The P2X7 receptor in infection and inflammation. Immunity. 2017:47(1):15-31.

56. Ahlroth MK, Kola EH, Kulomaa MS, Ewald D, Masabanda J, Sazanov A, et al. Characterization and chromosomal localization of the chicken avidin gene family. Anim Genet. 2000;31(6):367-75.

57. Tuohimaa P, Joensuu T, Isola J, Keinanen R, Kunnas T, Niemela A, et al. Development of progestin-specific response in the chicken oviduct. Int J Dev Biol. 1989;33(1):125-34.

58. Packialakshmi B, Liyanage R, Lay JO, Okimoto R, Rath NC. Proteomic changes in the plasma of broiler chickens with femoral head necrosis. Biomark Insights. 2016;11:55-62.

59. Lee J, Park C, Kim HJ, Lee YD, Lee ZH, Song YW, et al. Stimulation of osteoclast migration and bone resorption by C-C chemokine ligands 19 and 21. Exp Mol Med. 2017;49(7):e358. https://doi.org/10.1038/emm.2017.100.

60. Wideman RF, Al-Rubaye A, Kwon YM, Blankenship J, Lester H, Mitchell KN, et al. Prophylactic administration of a combined prebiotic and probiotic, or therapeutic administration of enrofloxacin, to reduce the incidence of bacterial chondronecrosis with osteomyelitis in broilers. Poult Sci. 2015;94(1):25-36.

61. Berendt T, Bch BM. Bone and joint infection. Clin Med (Northfield II). 2004; 4(6):510-8.

62. Wright JA, Nair SP. Interaction of staphylococci with bone. Int J Med Microbiol. 2010;300(2-3):193-204.

63. Heilmann C. Adhesion mechanisms of staphylococci. Adv Exp Med Biol. 2011;715:105-23.

64. Tucker KA, Reilly SS, Leslie CS, Hudson MC. Intracellular Staphylococcus aureus induces apoptosis in mouse osteoblasts. FEMS Microbiol Lett. 2000; 15:186(2):151-6.

65. Widaa A, Claro T, Foster TJ, O'Brien FJ, Kerrigan SW. Staphylococcus aureus protein A plays a critical role in mediating bone destruction and bone loss in Osteomyelitis. PLoS One. 2012;7(7):e40586. https://doi.org/10.1371/journal. pone.0040586.

66. Pinton P, Giorgi C, Siviero R, Zecchini E, Rizzuto R. Calcium and apoptosis: ER-mitochondria Ca2+ transfer in the control of apoptosis. Oncogene. 2008; 27(50):6407-18.

67. Humphreys BD, Rice J, Kertesy SB, Dubyak GR. Stress-activated protein kinase/JNK activation and apoptotic induction by the macrophage P2X7 nucleotide receptor. J Biol Chem. 2000;275(35):26792-8. 
68. Duchatelet S, Boyden LM, Ishida-Yamamoto A, Zhou J, Guibbal L, Hu R, et al. Mutations in PERP cause dominant and recessive Keratoderma. J Invest Dermatol. 2019 Feb 1;139(2):380-90.

69. Heckmann BL, Zhang X, Xie X, Liu J. The G0/G1 switch gene 2 (GOS2): regulating metabolism and beyond. Biochim Biophys Acta. 2013;1831(2):276-81.

70. Hardy R, Cooper MS. Bone loss in inflammatory disorders. J Endocrinol. 2009;201:309-20.

71. Miranda-Bautista J, Verdejo C, Díaz-Redondo A, Bretón I, Bellón JM, PérezValderas MD, et al. Metabolic bone disease in patients diagnosed with inflammatory bowel disease from Spain. Ther Adv Gastroenterol. 2019;12: 175628481986215.

72. Zhbannikov IY, Arbeev KG, Yashin Al. Rqt: an R package for gene-level metaanalysis. Bioinformatics. 2017;33(19):3129-30.

73. Li H. Durbin R. Bioinformatics: Fast and accurate short read alignment with Burrows-Wheeler transform; 2009:25(14):1754-60.

74. Anders S, Pyl PT, Huber W. HTSeq-A Python framework to work with highthroughput sequencing data. Bioinformatics. 2015;31(2):166-9.

75. Robinson MD, McCarthy DJ, Smyth GK. edgeR: a Bioconductor package for differential expression analysis of digital gene expression data. Bioinformatics. 2010;26(1):139-40.

76. Benjamini $Y$, Hochberg $Y$. Controlling the false discovery rate: a practical and powerful approach to multiple testing. J R Stat Soc Ser B. 1995;57(1): 289-300.

77. Conesa A, Götz S, García-Gómez JM, Terol J, Talón M, Robles M. Blast2GO: a universal tool for annotation, visualization and analysis in functional genomics research. Bioinformatics. 2005;21(18):3674-6.

78. Supek F, Bošnjak M, Škunca N, Šmuc T. Revigo summarizes and visualizes long lists of gene ontology terms. PLoS One. 2011;6(7):e21800. https://doi. org/10.1371/journal.pone.0021800.

79. Szklarczyk D, Franceschini A, Wyder S, Forslund K, Heller D, Huerta-Cepas J, et al. STRING v10: protein-protein interaction networks, integrated over the tree of life. Nucleic Acids Res. 2015;43(D1):D447-52.

80. Zhou G, Soufan O, Ewald J, Hancock REW, Basu N, Xia J. NetworkAnalyst 3.0 a visual analytics platform for comprehensive gene expression profiling and meta-analysis. Nucleic Acids Res. 2019;47(W1):W234-41.

81. Ye J, Coulouris G, Zaretskaya I, Cutcutache I, Rozen S, Madden TL. PrimerBLAST: a tool to design target-specific primers for polymerase chain reaction. BMC Bioinformatics. 2012;13:134. https://doi.org/10.1186/14712105-13-134.

82. Pfaffl MW, Horgan GW, Dempfle L. Relative expression software tool (REST) for group-wise comparison and statistical analysis of relative expression results in real-time PCR. Nucleic Acids Res. 2002;30(9):e36. https://doi.org/10. 1093/nar/30.9.e36

\section{Publisher's Note}

Springer Nature remains neutral with regard to jurisdictional claims in published maps and institutional affiliations.

Ready to submit your research? Choose BMC and benefit from:

- fast, convenient online submission

- thorough peer review by experienced researchers in your field

- rapid publication on acceptance

- support for research data, including large and complex data types

- gold Open Access which fosters wider collaboration and increased citations

- maximum visibility for your research: over $100 \mathrm{M}$ website views per year

At $\mathrm{BMC}$, research is always in progress.

Learn more biomedcentral.com/submissions 